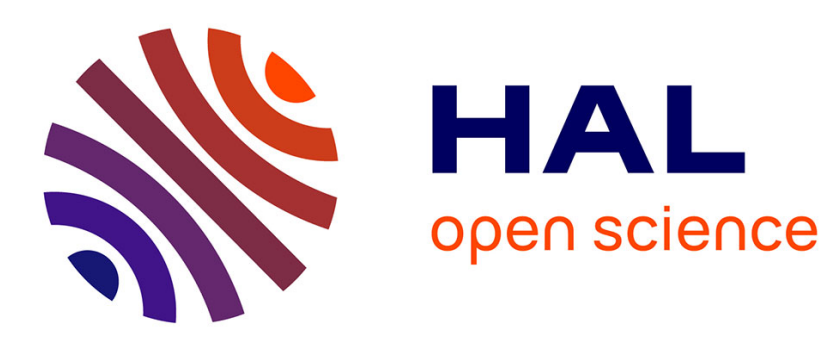

\title{
Determination and comparison of optimal eco-driving cycles for hybrid electric vehicles
}

\author{
Hippolyte Bouvier, Guillaume Colin, Yann Chamaillard
}

\section{To cite this version:}

Hippolyte Bouvier, Guillaume Colin, Yann Chamaillard. Determination and comparison of optimal eco-driving cycles for hybrid electric vehicles. 14th European Control Conference (ECC 2015), Jul 2015, Linz, Austria. pp.142-147. hal-01184913

\section{HAL Id: hal-01184913 \\ https://hal.science/hal-01184913}

Submitted on 18 Aug 2015

HAL is a multi-disciplinary open access archive for the deposit and dissemination of scientific research documents, whether they are published or not. The documents may come from teaching and research institutions in France or abroad, or from public or private research centers.
L'archive ouverte pluridisciplinaire HAL, est destinée au dépôt et à la diffusion de documents scientifiques de niveau recherche, publiés ou non, émanant des établissements d'enseignement et de recherche français ou étrangers, des laboratoires publics ou privés. 


\title{
Determination and comparison of optimal eco-driving cycles for hybrid electric vehicles
}

\author{
Hippolyte Bouvier, Guillaume Colin and Yann Chamaillard
}

\begin{abstract}
The aim of this work is, while using an optimal approach with dynamic programming (DP), to determine the best eco-driving cycle starting from a given driving cycle. To find the cycle with the minimum fuel consumption, two ways of computing an eco-driving cycle are compared. For a Hybrid Electric Vehicle (HEV), is it more efficient to compute an HEV based eco-driving cycle or to compute a conventional vehicle based eco-driving cycle and then to adapt it to a hybrid vehicle? Hence, two different DP programs are developed, that lead to two different eco-driving cycles: hybrid and conventional. Finally, the fuel consumption of an HEV with two Energy Management Strategies (EMS) will be compared: an off-line optimal one based on the Pontryagin Minimum Principle and an on-line suboptimal Equivalent Consumption Minimization Strategy (ECMS). The paper shows that in order to generate the best speed trajectory in terms of fuel consumption, it is necessary to take the fact that the vehicle is an HEV into account.
\end{abstract}

\section{INTRODUCTION}

For many years now, economic and ecological mutations have been driving changes in the main industrial sectors. The automotive sector is deeply concerned by these mutations as shown by the recent interest and extensive research in ecodriving strategies. Eco-driving could be defined as the way to generate a vehicle speed profile that reduces fuel consumption (and pollutant emissions) under some constraints (stop, distance, duration, etc.). The main bases of eco-driving come down to certain basic rules that anyone can respect: driving at a low engine speed, not at full throttle, anticipating the traffic, etc. With these rules, a driver can save $10 \%$ of fuel. For a better gain in fuel consumption, other methods such as computer simulation can be used [1], [2], [3]. These studies generally compute eco-driving cycles for conventional or full electric vehicles.

However, with two different sources of energy, the optimization of a hybrid eco-driving cycle is more complex [4]. The optimal interaction (or torque split) between the electric motor and the internal combustion engine (ICE) has to be determined during the calculation of the speed profile. [5] suggests a model that optimizes both the torque split and the speed profile using a Gradient method. More recent work [6], [7], combined Dynamic Programming (DP) with the energy management strategy (EMS) of a Hybrid Electric Vehicle (HEV) in order to obtain an eco-driving cycle. Lately, [8] worked on the direct optimization of EMS on hybrid vehicles with a view to reducing fuel consumption.

H. Bouvier, G. Colin and Y. Chamaillard are with Univ. Orléans, PRISME, EA 4229, F45072, Orléans, France. \{guillaume.colin, yann.chamaillard\}@univ-orleans.fr
In this work, the methodology comprises two main decoupled steps:

1) Determination of the eco-driving cycle with a 2Dimensional Dynamic Programming (section III).

2) Vehicle simulation on this cycle (section V) with an optimal or suboptimal EMS detailed in section IV.

In the first step, different optimal eco-driving cycles are computed using a 2-Dimensional Dynamic Programming (2D-DP) algorithm [9]. Three different cycles are obtained:

- the initial cycle: e.g. an Artemis cycle,

- the conventional eco-driving cycle: based on the initial cycle, a 2D DP generates a speed profile that minimizes the fuel consumption considering a conventional vehicle,

- the hybrid eco-driving cycle: based on the initial cycle, a 2D DP generates a speed profile that minimizes the fuel consumption considering an HEV.

Two models are therefore needed: the conventional vehicle and the HEV (section II). The motion equations are the same but the EMS is different. For the conventional vehicle, the EMS is straightforward because only one source of energy is taken into account. For the HEV model, the torque split has to be found for each second of the cycle [10] in order to minimize the fuel consumption on the whole cycle.

In the second step, the calculation of the torque split is made using an off-line optimal strategy based on the Pontryagin Minimum Principle, and an on-line suboptimal Equivalent Consumption Minimization Strategy (ECMS).

The paper is then organized as follows. In section II, the vehicle modeling is described. Dynamic programming principles with their algorithms are presented in section III, and the EMS are discussed in section IV. In the last section, results are given and compared for different cycles.

\section{VEHICLE MODELING}

\section{A. Motion equations}

The motion of the vehicle is the result of all the forces that are applied on it. The following model is quite simple and takes into account the main forces in a Galilean coordinate system, with Newton's second law of motion projected on an axis:

$$
m_{\text {veh }} \dot{v}_{v e h}=F_{\text {trac }}-F_{\text {res }}
$$

where $F_{\text {trac }}$ is the force generated by the powertrain, $F_{\text {res }}$ the sum of resistance forces, $m_{v e h}$ the vehicle mass and $v_{v e h}$ 
the vehicle speed. With the inertia of the vehicle $J_{v e h}=$ $m_{v e h} R_{w h e e l}^{2}$, the following relation can be written:

$J_{\text {veh }} \frac{\dot{v}_{\text {veh }}}{R_{\text {whel }}}=\left(F_{\text {trac }}-F_{\text {res }}\right) R_{\text {wheel }}=T_{\text {wheel }}-F_{\text {res }} R_{\text {weel }}$

The resistance forces comprise the rolling resistance force $F_{\text {rol }}(3)$, the aerodynamic drag force $F_{\text {aero }}(4)$ and a force due to road grade $F_{\text {grade }}(5)$.

$$
\begin{gathered}
F_{\text {rol }}=C_{r} m_{\text {veh }} g \cos (\alpha) \\
F_{\text {aero }}=0.5 \rho C_{d} A v_{\text {veh }}^{2} \\
F_{\text {grade }}=m_{\text {veh }} g \sin (\alpha)
\end{gathered}
$$

where $C_{r}$ is the rolling coefficient, $A$ the front area of the vehicle and $C_{d}$ the drag coefficient. As the constant road grade $\alpha$ is equal to zero, the third resistance force $F_{\text {grade }}$ is negligible.

The wheel torque $T_{\text {wheel }}$ can easily lead to the engine torque $T_{t o t}$, knowing the gear $u_{g b}$ and the differential characteristics:

$$
T_{w h e e l}=T_{t o t} \eta N_{f}\left(u_{g b}\right) N_{t}
$$

The model parameters are summed up in Table I.

TABLE I

VEHICLE MODEL PARAMETERS

\begin{tabular}{|llc|}
\hline Acronym & Description & Unit \\
\hline$m_{\text {veh }}$ & Vehicle mass & $\mathrm{kg}$ \\
$J_{\text {veh }}$ & Vehicle inertia & $\mathrm{kg} \cdot \mathrm{m}^{2}$ \\
$R_{\text {wheel }}$ & Wheel radius & $\mathrm{m}$ \\
$C_{r}$ & Rolling resistance coefficient & - \\
$g$ & Gravitational constant & $\mathrm{m} / \mathrm{s}^{2}$ \\
$\rho$ & Air density & $\mathrm{kg} / \mathrm{m}^{3}$ \\
$C_{d}$ & Vehicle's drag coefficient & - \\
$A$ & Vehicle's frontal surface & $\mathrm{m}^{2}$ \\
$\eta$ & Gear and differential efficiency & - \\
$N_{f}\left(u_{g b}\right)$ & Gearbox ratio & - \\
$N_{t}$ & Differential ratio & - \\
$\omega_{i d l e}$ & Engine idle speed & $\mathrm{rpm}$ \\
$R_{\text {int }}$ & Battery resistance & Ohm \\
$Q_{m a x}$ & Cell capacity & $C$ \\
$N b C e l l$ & Number of cells & - \\
$O C V$ & Open Circuit Voltage & $\mathrm{V}$ \\
\hline
\end{tabular}

By combining all the previous equations, a motion model giving the acceleration $\dot{v}_{v e h}$ as a function of the transmission torque $T_{\text {tot }}$ and the gear $u_{g b}$ can be written:

$$
\dot{v}_{v e h}=\frac{R_{w h e e l}}{J_{v e h}}\left\{\begin{array}{l}
T_{t o t} \eta N_{f}\left(u_{g b}\right) N_{t}- \\
R_{w h e e l}\left(C_{r} m_{v e h} g+0.5 \rho C_{d} A v_{v e h}^{2}\right)
\end{array}\right\}
$$

For an $\mathrm{HEV}$, the torque $T_{t o t}$ is a combination of the ICE Torque and the electric motor Torque. In this work, a parallel hybrid electric powertrain is considered.

Hence, the composition of the total torque $T_{t o t}$ is:

$$
T_{\text {tot }}=T_{\text {ice }}+R T_{\text {elec }}
$$

with $T_{i c e}=u_{T_{s}} T_{T o t}$ and $R T_{\text {elec }}=\left(1-u_{T_{s}}\right) T_{\text {tot }}$. Here, the transmission ratio between the electrical motor and the internal combustion engine $R$ is set to 0.5. Maximum and minimum authorized motor and ICE torques and speeds are considered in this model. Finally, the model gives the acceleration $\dot{v}_{v e h}$ as a function of the gear $u_{g b}$, the transmission torque $T_{\text {tot }}$ and the torque split $u_{T_{s}}$.

During braking phases, it is assumed that that as much energy as possible is recovered by the electric motor and lost by the ICE. The rest energy is dissipated by the friction braking system.

\section{B. Internal combustion engine (ICE)}

The ICE used for the tests is an engine with a maximum torque of 140 N.m for an engine speed of $2500 \mathrm{rpm}$ (red line in Fig. 1). Hence, with the speed of the wheels the engine speed can be computed:

$$
\begin{array}{r}
\omega_{\text {tot }}=\max \left(\omega_{\text {idle }}, \omega_{\text {wheel }} N_{f}\left(u_{g b}\right) N_{t}\right) \\
\omega_{\text {ice }}=\omega_{\text {tot }} \\
\omega_{\text {elec }}=\frac{\omega_{t o t}}{R}
\end{array}
$$

The fuel consumption $\dot{m}_{f}(\mathrm{~kg} / \mathrm{s})$ is computed through a lookup-table as a function of the engine speed and torque (Fig. 1).

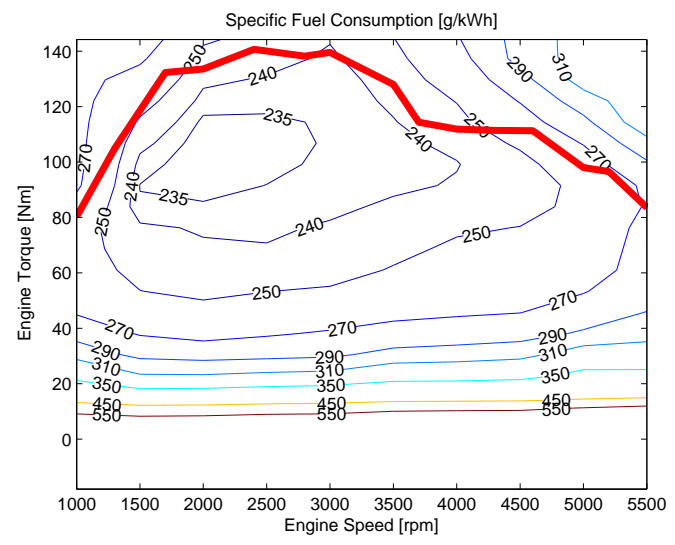

Fig. 1. Specific Fuel Consumption $(\mathrm{g} / \mathrm{kWh})$ of the internal combustion engine versus engine speed (rpm) and torque (Nm)

\section{Electric motor}

As for the ICE, the maximum torque given by the electric motor is known: 150 N.m for a motor speed of $2000 \mathrm{rpm}$ (red line in Fig. 2). The efficiency of the electric motor and the electric converter function of motor speed and torque are displayed on Fig. 2.

\section{Battery}

$P_{\text {elec }}$ is the power needed by the electric motor to propel the vehicle taken from the battery. Thanks to the Kirchhoff laws applied to this simplified model of the battery, the voltage and the current in the circuit were computed.

$$
\begin{array}{r}
U_{b a t}=\frac{1}{2} O C V+\frac{1}{2} \sqrt{O C V^{2}-4 P_{\text {elec }} R_{\text {int }}} \\
I_{\text {bat }}=\frac{O C V}{2 R_{\text {int }}}-\sqrt{\frac{O C V^{2}-4 P_{\text {elec }} R_{\text {int }}}{4 R_{\text {int }}^{2}}}
\end{array}
$$




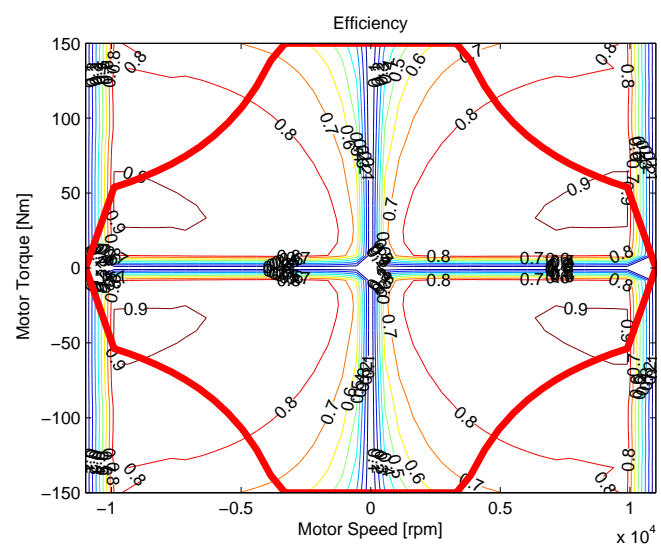

Fig. 2. Efficiency of the electric motor

where the Open Circuit Voltage $(O C V)$ is given by a lookup-table as a function of the State of Charge $(S O C)$, and the internal resistance $R_{\text {int }}$ is considered constant. The continuous evolution of the State Of Charge $(S O C)$ is given by:

$$
S O C(t)=\frac{1}{Q_{\max } N b C e l l} \int_{t_{0}}^{t}-I_{b a t t}(t) d t+\operatorname{SOC}\left(t_{0}\right)
$$

\section{ECO-DRIVING CYCLE COMPUTATION}

\section{A. Dynamic programming $(D P)$ principle}

In dynamic programming optimization, the search for the optimal trajectory is simplified using the Bellman principle while searching from the final state backward in time (or here in distance). The Bellman principle states the following: An optimal policy has the property that whatever the initial state and initial decision are, the remaining decisions must constitute an optimal policy with regard to the state resulting from the first decision [11].

Let us denote $V$ the minimum of the criterion $J=$ $\int_{t_{0}}^{t} L(\mathbf{u}(t), t) d t$ to minimize:

$$
V\left(\mathbf{x}_{t}, t\right):=\min J(\mathbf{x}(t), \mathbf{u}(t), t) .
$$

Bellman's principle states the following: let $t \in\left[t_{0}, t_{f}[\right.$ and $\mathbf{x}_{t} \in \mathbb{R}^{n}$ be given, then for all real $r \in\left[t, t_{f}\right]$

$$
V\left(\mathbf{x}_{t}, t\right)=\min _{\mathbf{u} \in \mathcal{U}}\left\{\int_{t}^{r} L(\mathbf{x}(\tau), \mathbf{u}(\tau), \tau) d \tau+V(\mathbf{x}(r), r)\right\}
$$

The structure of the algorithm is then simple. First, the problem is discretized or meshed: $\Delta t$ for the time $t_{k}$, $\Delta \mathbf{x}$ for the states $\mathbf{x}\left(t_{k}\right)=\mathbf{x}_{k}$ and $\Delta u$ for the inputs $\mathbf{u}_{k}$. Then, proceeding backward from the final state in $t_{f}$, the algorithm computes the cost from one state to another while the loop is in its initial state at $t_{0}$. Finally, the optimal saved control states are simulated in order to obtain the trajectory with the minimal cost (optimal trajectory).

Fig. 3 represents the principle of the DP algorithm for one state. The horizontal axis represents the time (or here the distance) and the vertical axis the state. Due to the state and time loops, the algorithm simulates the control sets (green ellipse) and saves only the optimal trajectory (red line) and puts the cost of the optimal trajectory to $V\left(\mathbf{x}_{k}, t_{k}\right)$.

Definition of the final cost $V\left(\mathbf{x}, t_{f}\right)$

- Start of time or spatial loop for $t_{k}=t_{f}:-\Delta t: t_{0}$

- Start of state loop for $\mathbf{x}_{k}=\mathbf{x}_{\min }: \Delta \mathbf{x}: \mathbf{x}_{\max }$

* Find $\mathbf{u}^{*}\left(\mathbf{x}_{k}, t_{k}\right)=\underset{\mathbf{u}_{k} \in U_{k}}{\arg \min }\left\{\Delta t L\left(\mathbf{x}_{k}, \mathbf{u}_{k}, t_{k}\right)+\right.$ $\left.V\left(\mathbf{x}_{k}+\Delta t f\left(\mathbf{x}_{k}, \mathbf{u}_{k}\right), t_{k}+1\right)\right\}$

- End of state loop

- End of time or spatial loop

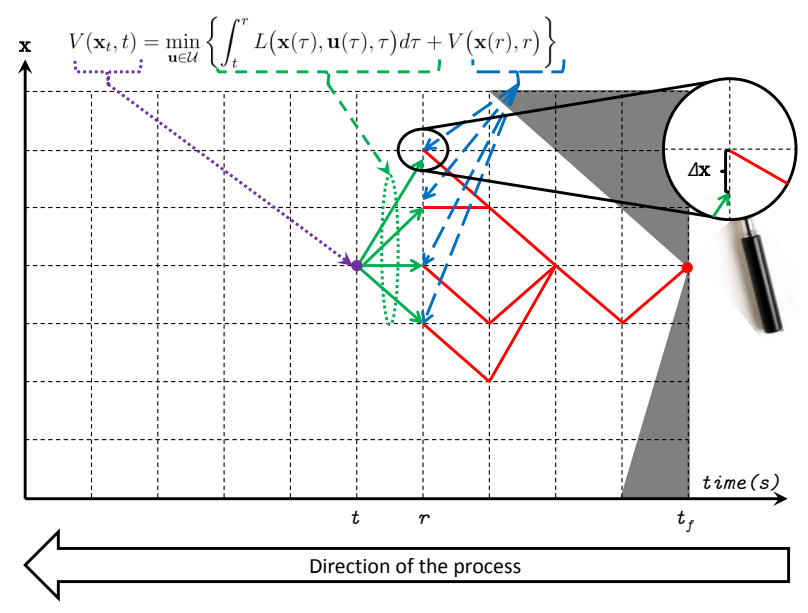

Fig. 3. Dynamic programming representation

The problem of dynamic programming is that the computation time increases with the number of states and control inputs. In our study, the distance $d$ and the vehicle speed $v$ are the two states, so that it is a 2D-DP. The control inputs are:

- the total torque delivered by the propulsion system $T_{t o t}$,

- the gear ratio $u_{g b}$,

- the torque split $u_{T_{s}}$ if the vehicle is an HEV.

\section{B. Methodology for eco-driving cycle computation}

To compute the eco-driving cycle, the initial driving cycle constraints [7] have to be included:

- vehicle speed limitations $(\mathrm{km} / \mathrm{h})$ : conventional European limitations $(0,30,50,70,80,90,100,110,130,160)$,

- the same distance $(\mathrm{m})$ and the same number of stops,

- almost the same duration (s).

Then, starting from an initial cycle, the goal is to find a new cycle with a lower fuel consumption that takes these constraints into account. For eco-driving cycle, vehicle stops are relevant in distance and not in time. The relationship between vehicle speed $v$, distance $s$ and time $t$ is:

$$
\frac{d v}{d s}=\frac{1}{v} \frac{d s}{d t}
$$


The discrete model of the vehicle is:

$$
\mathbf{x}_{k+1}=\left[\begin{array}{c}
v_{k+1} \\
d_{k+1}
\end{array}\right]=\left[\begin{array}{c}
f\left(v_{k}, \mathbf{u}_{k}\right) \\
d_{k}+\Delta d
\end{array}\right]
$$

where $f$ is the discrete form of equation (7).

Then, two cases are considered:

- Conventional vehicle eco-driving cycle

- HEV eco-driving cycle

1) Conventional eco-driving cycle:

In this case, the control input is $\mathbf{u}=\left[T_{t o t}, u_{g b}\right]$, and the cost function is:

$$
L\left(\mathbf{x}_{k}, \mathbf{u}_{k}\right)=\dot{m}_{f}\left(\mathbf{x}_{k}, \mathbf{u}_{k}\right)+\beta \Delta t+\text { Penalty }
$$

$\beta \Delta t$ is essential to constrain the trajectory in time, so $\beta$ is adjusted to obtain almost the same final time eco-driving cycle. The time could be added in state vector (18), but the computation time would be a problem [7]. The Penalty function penalizes the speeds that exceed the limits.

2) Hybrid eco-driving cycle:

In this case, the control input is $\mathbf{u}=\left[T_{t o t}, u_{g b}, u_{T_{s}}\right]$, and the cost function is:

$$
L\left(\mathbf{x}_{k}, \mathbf{u}_{k}\right)=\dot{m}_{f}\left(\mathbf{x}_{k}, \mathbf{u}_{k}\right)-\Lambda \Delta S O C+\beta \Delta t+\text { Penalty }
$$

As with the $\beta$ coefficient, the $\Lambda$ coefficient is adjusted to obtain a final SOC of $50 \%$. To compute $\triangle S O C$, the information on $O C V$ is needed. However, in backwards, i.e. beginning from the final state, no information about the battery $S O C$ is available. An average $O C V$ is therefore used here.

\section{ENERGY MANAGEMENT STRATEGY}

In an on-line Energy Management Strategy (EMS), it is necessary to find the best distribution between the electric path and the thermal path from the requested torque $T_{t o t}$ (generally deduced from the driver demand). The previous section generates an off-line strategy which is not realistic. In our case, it consists in finding the two control inputs $u_{g b}$, $u_{T_{s}}$ for a torque demand $T_{t o t}$. Most of the time, energy optimization in hybrid vehicles consists in minimizing the fuel consumption [12], [13]. Hence, energy management is a problem of optimal control with a finite horizon subject to system dynamics (generally the state of charge SOC) and final constraints (final $S O C$ ). Let $X \subset \mathbb{R}^{n}$ and $U \subset \mathbb{R}^{m}$ be the state and control sets. The $m$ control variables are denoted $\mathbf{u} \in U$ and the $n$ states denoted $\mathbf{x} \in X$. The optimal control problem consist in minimizing $J(\mathbf{x}, \mathbf{u}) \in \mathbb{R}$ with the following system dynamics:

$$
\dot{\mathbf{x}}=f(\mathbf{x}, \mathbf{u}),
$$

where $(\mathbf{x}, \mathbf{u}) \mapsto f(\mathbf{x}, \mathbf{u})$ is a function of class $\mathcal{C}^{1}$. Moreover, an initial condition is imposed $\mathbf{x}\left(t_{0}\right)=\mathbf{x}_{0}$.

Hence, the optimization problem can be summarized in:

$$
P_{0}(\mathbf{x}, \mathbf{u}):\left\{\begin{array}{cl}
\min _{\mathbf{u} \in U} J(\mathbf{x}, \mathbf{u}) & \\
\text { subject to } & \dot{\mathbf{x}}=f(\mathbf{x}, \mathbf{u}) \\
& \mathbf{x}\left(t_{0}\right)=\mathbf{x}_{0},
\end{array}\right.
$$

with a performance index to minimize:

$$
J(\mathbf{x}, \mathbf{u})=\Phi\left(\mathbf{x}\left(t_{f}\right)\right)+\int_{t_{0}}^{t_{f}} L(\mathbf{x}(t), \mathbf{u}(t), t) d t,
$$

where $\mathbf{x} \mapsto \Phi(\mathbf{x}(t))$ and $(\mathbf{x}, \mathbf{u}, t) \mapsto L(\mathbf{x}, \mathbf{u}, t)$ are of class $\mathcal{C}^{1}$. Generally, $L$ is the instantaneous fuel consumption and $J$ the total one.

In order to solve this problem it is necessary to know the whole trip. As the driving conditions are generally not known in advance, the theoretical optimal solution of problem (22) gives the achievable reference. Two methods have been developed to solve problem (22), a numerical solution called dynamic programming [14], [15], [10] and a analytical solution called equivalent dual problem [16], [10]. The second solution, which is very interesting for a real time use in a vehicle, is used here. It consists in defining a dual problem that has the same solution as the first one while putting the constraints and the cost function into a single function.

Let us define the Hamiltonian

$$
H(\mathbf{x}(t), \mathbf{u}(t), \boldsymbol{\lambda}(t), t)=L(\mathbf{x}(t), \mathbf{u}(t), t)+\boldsymbol{\lambda}(t)^{T} f(\mathbf{x}, \mathbf{u}),
$$

where $\boldsymbol{\lambda}$ is the Lagrange variable (or co-state) associated to the state $\mathbf{x}$. The resolution is based on the maximum principle stated by Lev Pontryagin [17] and the problem is simply solved by:

$$
\mathbf{u}^{*}(t)=\underset{\mathbf{u} \in U}{\arg \min } H\left(\mathbf{x}, \mathbf{u}, \boldsymbol{\lambda}^{*}, t\right)
$$

The main difficulty of this method is to obtain $\lambda^{*}$.

The Equivalent Consumption Minimization Strategy (ECMS) uses this principle of optimal control, while controlling the distribution between electrical energy and thermal energy by a penalty coefficient [10], [16], [18]. For a Hybrid Electric Vehicle, (24) can be rewritten into power flow, which is easier to interpret,

$$
H_{e q}(\mathbf{u}(t), \boldsymbol{\lambda}(t), t)=P_{f}(\mathbf{u}(t), t)+\boldsymbol{s}(t)^{T} P_{e}(\mathbf{u}, t),
$$

where

- $P_{f}(\mathbf{u}(t), t)=H_{L H V} \dot{m}_{f}(\mathbf{u}(t), t)$ is the thermal power with $H_{L H V}$ the lower heating value of the fuel and $\dot{m}_{f}(\mathbf{u}(t), t)$ the fuel flow,

- $P_{e}(\mathbf{u}, t)=-S \dot{O} C(t) \cdot O C V \cdot Q_{\max }$ is the battery electrical power with $O C V$ the Open Circuit Voltage and $Q_{\max }$ the nominal battery capacity.

The penalty coefficient $s(t)$, under the hypothesis of $\dot{\boldsymbol{\lambda}} \approx 0$, is often considered as a constant function of the driving cycle. In order to ensure charge sustaining operation, some authors propose to control this coefficient as a function of the State of Charge (SOC) [12], [19].

In this work, two strategies are considered in order to find the control inputs $u_{g b}$ and $u_{T_{s}}$ :

1) an off-line optimal strategy where $s(t)$ is determined by binary search in order to have $S O C\left(t_{f}\right)=50 \%$,

2) an on-line suboptimal strategy where $s(t)=s_{0}+$ $k_{p} e(t)+k_{i} \int_{t_{0}}^{t} e(t) d t . s_{0}$ is determined for the initial cycle and $e(t)=S O C_{r e f}-S O C(t)$. 


\section{RESULTS}

As previously explained, the simulations comprised two main steps:

1) Determination of the cycle: initial, ICE based ecodriving or HEV based eco-driving.

2) Computation on this cycle of the fuel consumption of the vehicle: Conventional, HEV with optimal EMS or HEV with suboptimal EMS.

To determine the eco-driving cycle, the following mesh was chosen: a spacing of $5 \mathrm{Nm}$ for $T_{t o t}$, of 1 for the gear box ratio $u_{g b}$ (from 1 to 5 ), of 0.2 for the torque split $u_{T_{s}}$ (from -1 to 1 ), of $\Delta d=1 \mathrm{~m}$ for the distance $d$, and of $0.2 \mathrm{~m} / \mathrm{s}$ for the vehicle speed $v$.

\section{A. Short urban Artemis cycle}

The algorithms were first tested on a short cycle, extracted from the Artemis Urban cycle. First, the initial driving cycle was computed versus distance and its maximum limits saved. Next, the 2D-DP was performed to generate the ecodriving cycles. The speed profiles are shown on Fig. 4 versus distance and on Fig. 5 versus time. Fig. 4 shows that the distances and the stops for each cycle are the same. Fig. 5 shows that the durations of the cycles are a little different, so the variable $\beta$ could be readjusted in order to have the same duration. Note that the duration of the stops was set to zero.

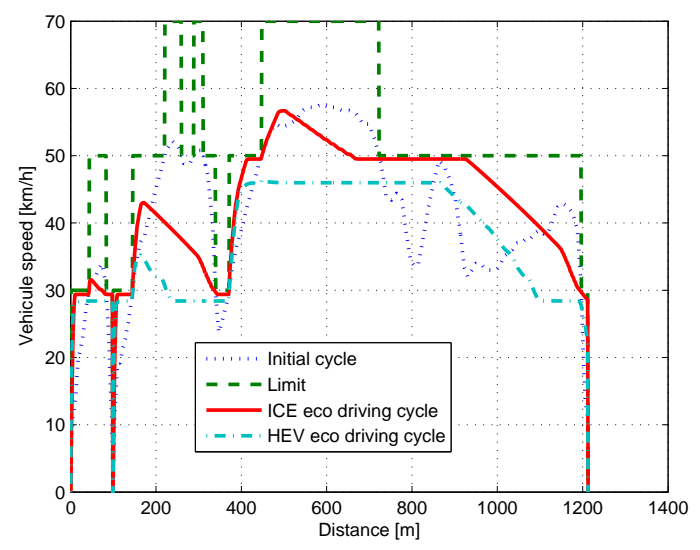

Fig. 4. Vehicle speed $(\mathrm{km} / \mathrm{h})$ vs distance (meter) for different cycles: initial (blue dotted line), Maximum authorized speed (green dashed line), Internal Combustion Engine based eco-driving (red solid line), Hybrid Electric Vehicle based eco-driving cycle (light blue line)

The fuel consumptions are compared in Table II. For the conventional vehicle, the minimum fuel consumption is obtained with the ICE based eco-driving cycle and for the HEV, the minimum fuel consumption is obtained with the HEV based eco-driving cycle and the optimal Energy Management Strategy (EMS). Simulations were performed with $k_{p}=0.1$ and $k_{i}=0.01$ to compute the penalty coefficient $s(t)$ of the suboptimal EMS. Note that readapting the EMS (here the constant $s_{0}$ ) is also important to reduce the fuel consumption of the vehicle on the driving cycle.

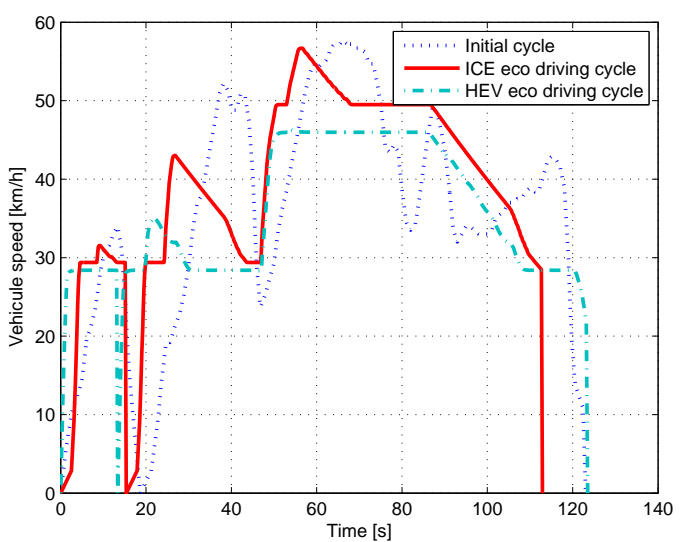

Fig. 5. Vehicle speed $(\mathrm{km} / \mathrm{h})$ vs time (s) for different cycles: initial (blue dotted line), Internal Combustion Engine based eco-driving (red solid line), Hybrid Electric Vehicle based eco-driving cycle (light blue line)

TABLE II

CORRECTED FuEL CONSUMPTION (L/100KM) OBTAINED FOR THE THREE DIFFERENT CYCLES FOR THREE VEHICLES BASED ON A SHORT URBAN ARTEMIS CYCLE

\begin{tabular}{|l|ccc|}
\hline & Initial Cycle & ICE eco cycle & HEV eco cycle \\
\hline Conv. vehicle & 6.99 & 4.74 & 4.84 \\
HEV opt EMS & 4.55 & 3.82 & 3.69 \\
HEV subopt EMS & 4.61 & 3.83 & 3.75 \\
\hline
\end{tabular}

\section{B. Worldwide harmonized Light vehicles Test Cycle}

The algorithms were then tested on the Worldwide harmonized Light vehicles Test Cycle (WLTC). The same methodology as in section V-A was used. The speed profiles are shown on Fig. 6 versus distance and Fig. 7 versus time. Fig. 6 shows that the distances and the stops for each cycle are the same.

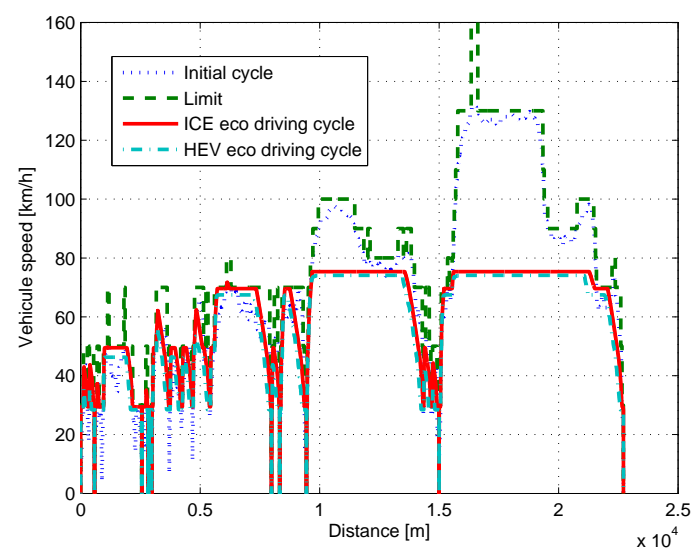

Fig. 6. Vehicle speed $(\mathrm{km} / \mathrm{h})$ vs distance $(\mathrm{m})$ for different cycles: WLTC (blue dotted line), Maximum authorized speed (green dashed line), Internal Combustion Engine based eco-driving (red solid line), Hybrid Electric Vehicle based eco-driving cycle (light blue line)

The fuel consumptions are compared in Table III for the 


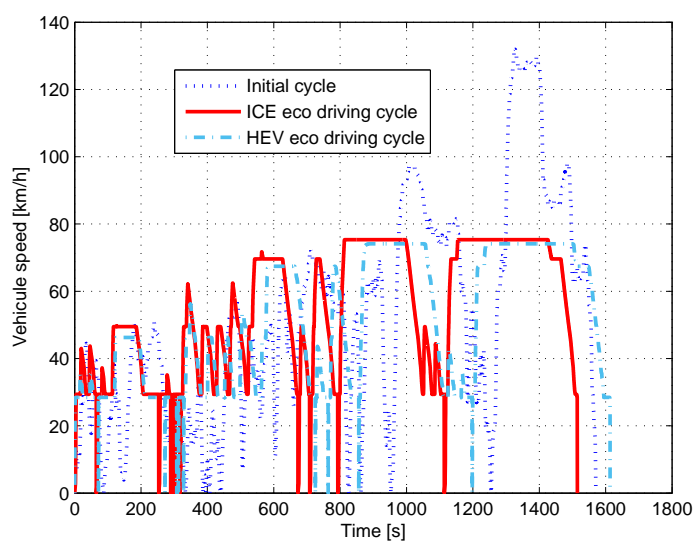

Fig. 7. Vehicle speed $(\mathrm{km} / \mathrm{h})$ vs time (s) for different cycles: WLTC (blue dotted line), Internal Combustion Engine based eco-driving (red solid line), Hybrid Electric Vehicle based eco-driving cycle (light blue line)

WLTC. As in section V-A, the minimum fuel consumption was obtained with the ICE based eco-driving cycle for the conventional vehicle and with the HEV based ecodriving cycle and the optimal EMS for the HEV. The same conclusions can be drawn with the WLTC as with the short Artemis urban cycle. On this example, the fuel consumption of a conventional car with eco-driving is lower than the fuel consumption of an HEV without eco-driving. Unsurprisingly, combining eco-driving and HEV gives the best results.

TABLE III

CORRECTED FUEL CONSUMPTION (L/100KM) OBTAINED FOR THE THREE DIFFERENT CYCLES FOR THREE VEHICLES BASED ON THE WLTC

\begin{tabular}{|l|ccc|}
\hline & Initial Cycle & ICE eco cycle & HEV eco cycle \\
\hline Conv. vehicle & 6.51 & 4.91 & 4.96 \\
HEV opt EMS & 5.08 & 4.17 & 4.06 \\
HEV subopt EMS & 5.23 & 4.22 & 4.10 \\
\hline
\end{tabular}

For the WLTC, eco-driving decreases the fuel consumption by $16 \%$ (ICE based) to $19 \%$ (HEV based) for an HEV. Hence, taking into account the fact the vehicle is an HEV in the generation of the eco-cycle saves from 2 to $3 \%$ of fuel for both cycles. Finally, readapting the strategy (i.e. $s(t)$ of (26)) function of the eco-driving cycle saves from $1 \%$ to $2 \%$ of fuel.

\section{CONCLUSIONS}

In this paper, an optimal approach using dynamic programming was used to determine the best eco-driving cycle starting from a given driving cycle for a conventional car and a Hybrid Electric Vehicle. The results show that the minimal fuel consumption is obtained when combining ecodriving and HEV. To generate the best speed trajectory in terms of fuel consumption, it is necessary to take the fact that the vehicle is a Hybrid Electric Vehicle into account, although eco-driving behavior is more important than the way in which the eco cycle is computed (considering that the vehicle is hybrid or not). Moreover, readapting the Energy
Management Strategy function of the eco-driving cycle also reduces the fuel consumption of the vehicle.

Finally, whatever the chosen strategy for generating the cycle, eco-driving with a conventional car saves as much fuel as with an HEV. However, as eco-driving depends on the acceptability of the driver while HEV does not, ecodriving could be seen as a potential gain and HEV as a real gain. With autonomous cars, this potential gain will become a real one.

\section{REFERENCES}

[1] F. Mensing, R. Trigui, and E. Bideaux, "Vehicle trajectory optimization for application in eco-driving," in Vehicle Power and Propulsion Conference (VPPC), 2011 IEEE, Sept 2011, pp. 1-6.

[2] W. Dib, A. Chasse, P. Moulin, A. Sciarretta, and G. Corde, "Optimal energy management for an electric vehicle in eco-driving applications," Control Engineering Practice, vol. 29, pp. 299-307, 2014.

[3] H. T. Luu, L. Nouveliere, and S. Mammar, "Dynamic programming for fuel consumption optimization on light vehicle," in Advances in Automotive Control, 2010, pp. 372-377.

[4] T. van Keulen, B. Jager, D. Foster, and M. Steinbuch, "Velocity trajectory optimization in hybrid electric trucks," in Proceeding of American Control Conference, July 2010.

[5] T. S. Kim, C. Manzie, and R. Sharma, "Model predictive control of velocity and torque split in a parallel hybrid vehicle," in Systems, Man and Cybernetics, 2009. SMC 2009. IEEE International Conference on. IEEE, 2009, pp. 2014-2019.

[6] F. Mensing, E. Bideaux, R. Trigui, and H. Tattegrain, "Trajectory optimization for eco-driving taking into account traffic constraints," Transportation Research Part D: Transport and Environment, vol. 18, pp. 55-61, 2013.

[7] F. Mensing, E. Bideaux, R. Trigui, J. Ribet, and B. Jeanneret, "Ecodriving: An economic or ecologic driving style?" Transportation Research Part C: Emerging Technologies, vol. 38, pp. 110-121, 2014.

[8] G. Heppeler, M. Sonntag, and O. Sawodny, "Fuel efficiency analysis for simultaneous optimization of the velocity trajectory and the energy management in hybrid electric vehicles," in 19th IFAC World Congress, IFAC'14, 2014.

[9] D. Bertsekas, Dynamic programming and optimal control. Athenas scientific, 2000

[10] L. Guzzella and A. Sciarretta, Vehicle propulsion systems: Introduction to modeling and optimization. Springer, 2005.

[11] R. Bellman, Dynamic programming. Princeton University press, Princeton, NJ, 1957.

[12] A. Sciarretta, M. Back, and L. Guzzella, "Optimal control of parallel hybrid electric vehicles," IEEE Transactions on control systems technology, vol. 12, no. 3, pp. 352-363, 2004.

[13] G. Rousseau, A. Sciarretta, and D. Sinoquet, "Optimal energy management of a mild-hybrid vehicles," 4th European conference on alternative energies for the automotive industry, 2008.

[14] O. Sundström, L. Guzzella, and P. Soltic, "Optimal hybridization in two parallel hybrid electric vehicles using dynamic programming," Proceedings of the 17th World IFAC Congress, Korea, 2008.

[15] C.-C. Lin, J.-M. Kang, J. Grizzle, and H. Peng, "Energy management strategy for a parallel hybrid electric truck," in American Control Conference, vol. 4, 2001, pp. 2878-2883.

[16] S. Delprat, J. Laubert, T. Guerra, and J. Rimaux, "Control of a parallel hybrid powertrain: optimal control," IEEE Transactions on Vehicular technology, vol. 53, no. 3, pp. 872-881, 2004.

[17] L. S. Pontryagin, V. Boltyanskii, R. Gamkrelidze, and E. Mischchenko, "The mathematical theory of optimal processes, Interscience, New York," 1962.

[18] A. Sciarretta, L. Guzzella, and M. Back, "A real-time optimal control statement for parallel hybrid vehicles with on-board estimation of control parameters," Proceedings of IFAC symposium advance in automotive control, pp. 502-507, 2004.

[19] A. Chasse, P. Pognant-gros, and A. Sciarretta, "Online implementation of an optimal supervisory control for a parallel hybrid powertrain," SAE technical paper, vol. 2009-01-1868, 2009. 